\title{
LEAST REGRET CONTROL, VIRTUAL CONTROL AND DECOMPOSITION METHODS*
}

\author{
JACQUES-LOUIS LIONS ${ }^{1}$
}

\begin{abstract}
Least regret control" consists in trying to find a control which "optimizes the situation" with the constraint of not making things too worse with respect to a known reference control, in presence of more or less significant perturbations. This notion was introduced in [7]. It is recalled on a simple example (an elliptic system, with distributed control and boundary perturbation) in Section 2. We show that the problem reduces to a standard optimal control problem for augmented state equations. On another hand, we have introduced in recent notes [9-12] the method of virtual control, aimed at the "decomposition of everything" (decomposition of the domain, of the operator, etc). An introduction to this method is presented, without a priori knowledge needed, in Sections 3 and 4, directly on the augmented state equations. For problems without control, or with "standard" control, numerical applications of the virtual control ideas have been given in the notes [9-12] and in the note [5]. One of the first systematic paper devoted to all kind of decomposition methods, including multicriteria, is a joint paper with A. Bensoussan and R. Temam, to whom this paper is dedicated, $c f .[1]$.
\end{abstract}

Mathematics Subject Classification. 49A22, 35J99.

Received: October 18, 1999.

\section{INTRODUCTION}

\subsection{Least regret control}

Let us first recall what "least regret control" is all about. We present it on the simplest possible example.

Let $\Omega$ be a (bounded) open set in $\mathbb{R}^{d}(d=2,3$ in most of the applications), with (smooth) boundary $\partial \Omega=\Gamma$. In $\Omega$ we are given a second order elliptic operator

$$
A \varphi=-\sum_{i, j=1}^{d} \frac{\partial}{\partial x_{i}}\left(a_{i j}(x) \frac{\partial \varphi}{\partial x_{j}}\right)+a_{0} \varphi
$$

Keywords and phrases. Control, least regret, domain decomposition, virtuel control.

* Dedicated to Roger Teman for his 60th birthday

${ }^{1}$ Collège de France, 3 rue d'Ulm, 75231 Paris Cedex 05, France. 
where $a_{0}, a_{i, j} \in L^{\infty}(\Omega)$ satisfy a.e.

$$
\sum_{i, j=1}^{d} a_{i j}(x) \zeta_{i} \zeta_{j} \geq c \sum_{i=1}^{d} \zeta_{i}^{2}, \quad a_{0} \geq c, \quad c>0 .
$$

The state $z$ of the equation is given by the solution of

$$
\begin{aligned}
A z & =v 1_{\mathcal{O}} \quad \text { in } \Omega, \\
\frac{\partial z}{\partial n_{A}} & =g \quad \text { on } \Gamma
\end{aligned}
$$

where

$$
\mid \begin{aligned}
& \mathcal{O}=\text { open set contained in } \Omega, v \in L^{2}(\mathcal{O}), v=\text { control variable, } \\
& 1_{\mathcal{O}}=\text { characteristic function of } \mathcal{O} \\
& \frac{\partial}{\partial n_{A}}=\text { conormal derivative with respect to } A, \\
& g \in L^{2}(\Gamma), g=\text { perturbation (or unknown) variable. }
\end{aligned}
$$

(Condition (1.3) is taken through a weak variational formulation).

Problem (1.2)-(1.3) admits a unique solution:

$$
z=z(v, g) \in H^{1}(\Omega) .
$$

We then introduce the cost function

$$
J(v, g)=\frac{1}{2} \int_{\Omega} \rho(x)\left(z(v, g)-z_{0}\right)^{2} \mathrm{~d} x+\frac{1}{2} \int_{\mathcal{O}} v^{2} \mathrm{~d} x
$$

where $\rho$ is given in $L^{\infty}(\Omega), \rho \geq 0$, and where $z_{0}$ is the "optimal" state we wish to get close to, taking into account the "cost of the control" (expressed by the term $\frac{1}{2} \int_{\mathcal{O}} v^{2} \mathrm{~d} x$ in (1.6)).

If $g$ is known, say $g=g_{0}, J(v, g)$ does not depend on $g$, and the problem we wish to solve is to find

$$
\inf _{v} J\left(v, g_{0}\right) \text {. }
$$

This is a standard problem of optimal control for distributed systems, in one of the simplest possible case. Cf. [6].

But here we have a perturbation on the boundary, expressed by $g$. We assume that, by tradition or by formal computation, one is used to apply a "nominal policy", i.e. that one uses

$$
v=v_{0} \text { given in } L^{2}(\mathcal{O}) \text {. }
$$

We want to choose $v$ in the best possible way with respect to (1.6), with the natural constraint that we do not want to deteriorate the situation with respect to the traditional policy $v=v_{0}$. Analytically, this is expressed by the problem to find

$$
\inf _{v} \sup _{g}\left[J(v, g)-J\left(v_{0}, g\right)\right]
$$


If there is a solution, it is called "policy without regret". But (1.8) is too restrictive. There is no solution of (1.8) in general. Hence the introduction of the following (relaxed) problem

$$
\inf _{v} \sup _{g}\left[J(v, g)-J\left(v_{0}, g\right)-\frac{\gamma}{2} \int_{\Gamma} g^{2} \mathrm{~d} \Gamma\right]
$$

where $\gamma$ is $>0$ and "small".

Problem (1.9) admits (in the present situation) a unique solution $v$ (see below in Sect. 2) which is called "policy with least regret", or "least regret control".

Remark 1.1. The notions of "without regret control" or "least regret control" have been introduced in [7].

The notion of regret was first introduced by [13] (a reference indicated to me by D. Gabay, after publication of the note [7]).

The notion of "least regret control" is completely general and immediately extends to evolution problems and non linear systems.

It has be extended in [3] to multi criteria and multi agents.

Our goal here is to study (1.9) with the state given by (1.2) (1.3), with the cost function given by (1.6), in the framework of Decomposition Methods.

\subsection{Decomposition methods}

Given any problem involving a partial differential operator $A$ (of any type) in a domain $\Omega$, an important question is to decompose $A$ and, or, the domain $\Omega$, so as to "cut the problem in a large number of small and simple pieces" (with parallelism in sight).

A huge amount of work is devoted to these methods (no attempt is made for a significant bibliography). A systematic paper was devoted to these questions, namely [1] (a paper which seems to have been forgotten, including by his authors...).

In a series of notes [9-12] we have introduced the technique of virtual control for "the decomposition of everything" (domain decomposition, decomposition of operator), applied in [5] to decomposition of the "energy space".

Our goal is to show here that these techniques can be applied to least regret controls problems.

In order not to snow the ideas under complicated technicalities we present the virtual control technique in Section 3 for the problem (1.9) in a single preliminary framework, somewhat connected with fictitious domains, further extensions being briefly indicated in Section 4.

We now proceed with (1.9) and the introduction of the augmented state equations.

\section{Augmented system}

\subsection{Preliminary computations}

Let us introduce the states $y=y(v)$ and $\varphi(g)$ defined by

$$
\begin{aligned}
& A y=v 1_{\mathcal{O}} \text { in } \Omega, \frac{\partial y}{\partial n_{A}}=0 \text { on } \Gamma, \\
& A \varphi=0 \text { in } \Omega, \frac{\partial \varphi}{\partial n_{A}}=g \text { on } \Gamma
\end{aligned}
$$


so that

$$
z(v, g)=y(v)+\varphi(g)
$$

Moreover in order to (slightly) simplify the exposition and without restriction (in linear problems) we assume that

$$
v_{0}=0 .
$$

Then

$$
J(v, g)-J\left(v_{0}, g\right)=\frac{1}{2} \int_{\Gamma} \rho\left(y-z_{0}\right)^{2} \mathrm{~d} \Gamma-\frac{1}{2} \int_{\Gamma} \rho z_{0}^{2} \mathrm{~d} \Gamma+\int_{\Gamma} \rho y \varphi \mathrm{d} \Gamma
$$

(where we have written $y$ for $y(v), \varphi$ for $\varphi(g)$ ), or

$$
J(v, g)-J\left(v_{0}, g\right)=J(v, 0)-J\left(v_{0}, 0\right)+\int_{\Gamma} \rho y \varphi \mathrm{d} \Gamma .
$$

We then introduce the function $\eta$ defined by

$$
A^{*} \eta=0 \text { in } \Omega, \quad \frac{\partial \eta}{\partial n_{A^{*}}}=\rho y \text { on } \Gamma,
$$

where $A^{*}$ denotes the adjoint of $A$.

The set $\{y, \eta\}$ given by (2.1) (2.6) is the augmented state.

Using (2.6) one has

$$
\int_{\Gamma} \rho y \varphi \mathrm{d} \Gamma=\int_{\Gamma} \eta g \mathrm{~d} \Gamma
$$

so that

$$
J(v, g)-J\left(v_{0}, g\right)-\frac{\gamma}{2} \int_{\Gamma} g^{2} \mathrm{~d} \Gamma=J(v, 0)-J\left(v_{0}, 0\right)+\int_{\Gamma} \eta g \mathrm{~d} \Gamma-\frac{\gamma}{2} \int_{\Gamma} g^{2} \mathrm{~d} \Gamma .
$$

Therefore

$$
\mid \begin{aligned}
& \sup \cdot\left[J(v, g)-J\left(v_{0}, g\right)-\frac{\gamma}{2} \int_{\Gamma} g^{2} \mathrm{~d} \Gamma\right]= \\
& =J(v, 0)-J\left(v_{0}, 0\right)+\frac{1}{2 \gamma} \int_{\Gamma} \eta^{2} \mathrm{~d} \Gamma .
\end{aligned}
$$

We now have to minimize the expression in (2.8). Of course $J\left(v_{0}, 0\right)$ is fixed. Therefore the problem reduces to

$$
\inf _{v} \cdot\left[J(v, 0)+\frac{1}{2 \gamma} \int_{\Gamma} \eta^{2} \mathrm{~d} \Gamma\right]
$$

where $\eta=\eta(v)$, the state $\{y(v), \eta(v)\}$ being given by the solution of the augmented system (2.1) (2.6).

A few remarks are now in order. 


\subsection{Remarks}

Remark 2.1. Problem (2.9) is now a standard problem of optimal control for a distributed system, when the state equation is a set of two elliptic equations (2.1)-(2.6) (coupled by the boundary condition $\frac{\partial \eta}{\partial n_{A^{*}}}=\rho y$ ). It is therefore "normal" that the general methods of virtual control apply to the present situation! We simply show in Section 3 how the methods of O. Pironneau and the A. already referred to can be adapted to the present situation.

Remark 2.2. One can also view "least regret" as a way to "increase the robustness" of the control, subject to perturbations on the boundary.

Remark 2.3. Let us assume that we do have some information on the perturbation $g$, expressed by

$$
g \in G=\text { closed convex subset of } L^{2}(\Gamma) .
$$

Then of course (2.9) is replaced by

$$
\inf _{v} \cdot\left[J(v, 0)+\sup _{g \in G}\left(\int_{\Gamma} \eta g \mathrm{~d} \Gamma-\frac{\gamma}{2} \int_{\Gamma} g^{2} \mathrm{~d} \Gamma\right)\right] .
$$

Section 3 can be applied to (2.11).

Remark 2.4. We can see in the above situation why the introduction of $\gamma>0$ is necessary (in general). Indeed if $\gamma=0$ (control without regret), one should have $\eta=0$ on $\Gamma$. Therefore (2.6) implies that $\eta=0$ in $\Omega$, so that $y=0$ on $\Gamma$ and the problem amounts to finding

$$
\inf \frac{1}{2} \int_{\mathcal{O}} v^{2} \mathrm{~d} x
$$

for all $v$ 's such that

$$
A y=v 1_{\mathcal{O}}, y=0 \text { and } \frac{\partial y}{\partial n_{A}}=0 \text { on } \Gamma
$$

the solution being $v=0$ !

Remark 2.5. For non linear state equations, the decomposition (2.3) is of course not valid. One then replaces $J(v, g)$ by

$$
J(v)+\left\langle\frac{\partial J}{\partial g}(v, g), g\right\rangle
$$

if the state equation is differentiable with respect to $g$, and which makes sense in case (2.10) with $G$ "small".

We now introduce a virtual control technique.

\section{ViRTUAL CONTROLS}

\subsection{Embedding and virtual controls}

We embed $\Omega$ (which can have a "complicated" boundary) into a large set $\tilde{\Omega}$ (say a cube, or a sphere) and we introduce a new system in $\tilde{\Omega}$. It is an "extension to $\tilde{\Omega}$ " of the augmented system introduced in Section 2.1. Let $\omega$ and $\omega_{*}$ be two open sets contained in $\tilde{\Omega} / \bar{\Omega}$ (cf. Fig. 1).

Let $\tilde{A}$ denote any extension of $A$ into $\tilde{\Omega}$, $\tilde{A}$ being elliptic strictly coercive in $\tilde{\Omega}$ (this is possible!). 


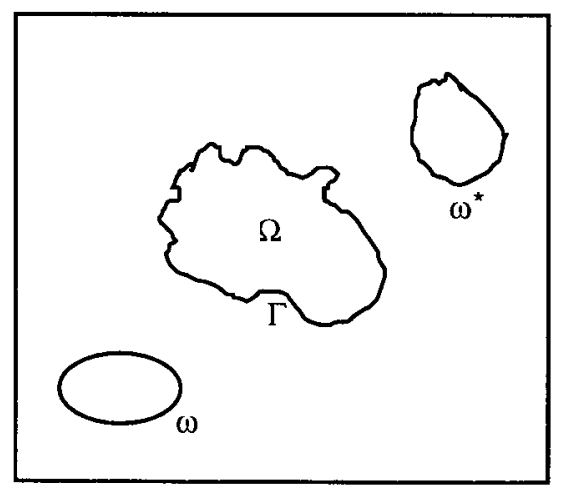

FiguRe 1

We now introduce the following system:

$$
\mid \begin{aligned}
& \tilde{A} \tilde{y}=v 1_{\mathcal{O}}+\lambda \chi, \\
& \tilde{A}^{*} \tilde{\eta}=\mu \chi_{*}, \\
& \tilde{y}, \tilde{\eta} \text { subject to any "simple" boundary condition on } \partial \tilde{\Omega}
\end{aligned}
$$

(for instance we can make the coefficients of $\tilde{A}$ periodic in $\tilde{\Omega}$ and take periodic boundary conditions). In (3.1) $\chi$ (resp. $\chi_{*}$ ) denotes the characteristic function of $\omega$ (resp. $\omega_{*}$ ), and $\lambda$ and $\mu$ are (for the time being) arbitrary functions,

$$
\lambda \in L^{2}(\omega), \mu \in L^{2}\left(\omega_{*}\right) .
$$

They are the virtual control.

Remark 3.1. Of course the restrictions of $\tilde{y}, \tilde{\eta}$ to $\Omega$, say $y, \eta$, satisfy $A y=v 1_{\mathcal{O}}, A^{*} \eta=0$ in $\Omega$, but do not satisfy in general the boundary conditions for $y$ and $\eta$ ! The virtual controls have precisely to be chosen in such a way that these boundary conditions are satisfied, at least approximately. This is possible, as we now show.

Given $v \in L^{2}(\mathcal{O})$, it is possible to choose $\lambda \in L^{2}(\omega)$ and $\mu \in L^{2}\left(\omega_{*}\right)$ in such a way that

$$
\mid \begin{aligned}
& \left|\frac{\partial \tilde{y}}{\partial n_{A}}\right|_{L^{2}(\Gamma)} \quad \text { and }\left|\frac{\partial \eta}{\partial n_{A^{*}}}-\rho \tilde{y}\right|_{L^{2}(\Gamma)} \\
& \text { are arbitrarily small. }
\end{aligned}
$$

Indeed, let us consider the mapping

$$
\lambda, \mu \rightarrow \frac{\partial \tilde{y}}{\partial n_{A}}, \frac{\partial \tilde{\eta}}{\partial n_{A^{*}}}-\rho \tilde{y}
$$

from $L^{2}(\omega) \times L^{2}\left(\omega^{*}\right) \rightarrow L^{2}(\Gamma) \times L^{2}(\Gamma)$.

Its range is dense.

Indeed one shows first (by duality and a unique continuation argument) that the range of $\lambda \rightarrow \frac{\partial \tilde{y}}{\partial n_{A}}$ is dense in $L^{2}(\Gamma)\left(\tilde{y}\right.$ depends only on $\lambda$, once $v$ is fixed). Then it suffices to show that $\mu \rightarrow \frac{\partial \tilde{\eta}}{\partial n_{A^{*}}}$ has a dense range in $L^{2}(\Gamma)$ (which is exactly the same result than for $\tilde{y}$ ). 
If $\lambda$ and $\mu$ are chosen according to (3.3), problem (2.9) is approximated (as closely as we want) by

$$
\inf _{v} \tilde{\mathcal{J}}(v)
$$

where

$$
\tilde{\mathcal{J}}(v)=\frac{1}{2} \int_{\Gamma} \rho\left(\tilde{y}-z_{0}\right)^{2} \mathrm{~d} \Gamma+\frac{1}{2} \int_{\mathcal{O}} v^{2} \mathrm{~d} x+\frac{1}{2 \gamma} \int_{\Gamma}(\tilde{\eta})^{2} \mathrm{~d} \Gamma
$$

In order to proceed one penalizes the conditions (3.3). We introduce

$$
\tilde{\mathcal{J}}(v, \lambda, \mu)=\tilde{\mathcal{J}}(v)+\frac{1}{2 \varepsilon} \int_{\Gamma}\left(\frac{\partial \tilde{y}}{\partial n_{A}}\right)^{2} \mathrm{~d} \Gamma+\frac{1}{2 \varepsilon} \int_{\Gamma}\left(\frac{\partial \tilde{\eta}}{\partial n_{A^{*}}}-\rho y\right)^{2} \mathrm{~d} \Gamma
$$

where $\varepsilon$ is fixed "small". Then an approximation of the least regret control problem considered here, is given by the solution of the problem.

$$
\inf _{v, \lambda, \mu} \tilde{\mathcal{J}}(v, \lambda, \mu)
$$

Remark 3.2. Problem (3.8) certainly looks more complicated than the formulation (2.9)! But

1. the domain $\tilde{\Omega}$ is chosen to be much simpler than $\Omega$;

2. the method presented here can be thought of as an introduction to domain decomposition for least regret control problems.

Remark 3.3. Let's suppose we shall be happy with the (small) errors $\left|\frac{\partial \tilde{y}}{\partial n_{A}}\right|_{L^{2}(\Gamma)} \leq \varepsilon_{1},\left|\frac{\partial \tilde{\eta}}{\partial n_{A^{*}}}-\rho \tilde{y}\right|_{L^{2}(\Gamma)} \leq \varepsilon_{1}$. One has then to choose $\varepsilon$ in (3.7) so that these conditions are (approximately) satisfied. This can be made more precise by transforming (3.7) by a duality argument (based on Fenchel-Rockafellar duality theorem, $c f$. for instance [2]), as it is used in a different situation by [4].

Assuming that $\varepsilon$ has been chosen in (3.7), we now give a simple algorithm of approximation of (3.8).

\subsection{Algorithm}

We do not use in this section the state $y, \eta$ given by (2.1) (2.6). Therefore we drop here the symbols $\sim$ in equation (3.1) and in the functional (3.7).

The first variation of $\mathcal{J}(v, \lambda, \mu)$ is given by

$$
\begin{aligned}
\delta \mathcal{J}(v, \lambda, \mu)= & \int_{\Gamma} \rho\left(y-z_{0}\right) \delta y+\int_{\mathcal{O}} v \delta v+\frac{1}{\gamma} \int_{\Gamma} \eta \delta \eta \\
& +\frac{1}{\varepsilon} \int_{\Gamma} \frac{\partial y}{\partial n_{A}} \frac{\partial \delta y}{\partial n_{A}}+\frac{1}{\varepsilon} \int_{\Gamma}\left(\frac{\partial \eta}{\partial n_{A^{*}}}-\rho y\right)\left(\frac{\partial \delta y}{\partial n_{A^{*}}}-\rho \delta y\right)
\end{aligned}
$$

where we skip the surface and volume elements $\mathrm{d} \Gamma$ and $\mathrm{d} x$.

We introduce now the adjoint functions $p$ and $\pi$ defined as follows:

$$
\int_{\tilde{\Omega}} p \tilde{A} \varphi=\int_{\Gamma} \rho\left(y-z_{0}\right) \varphi+\frac{1}{\varepsilon} \int_{\Gamma} \frac{\partial y}{\partial n_{A}} \frac{\partial \varphi}{\partial n_{A}}-\frac{1}{\varepsilon} \int_{\Gamma}\left(\frac{\partial \eta}{\partial n_{A^{*}}}-\rho y\right) \rho \varphi .
$$


$\forall \varphi \in H^{2}(\tilde{\Omega})$, satisfying the approximate boundary conditions on $\partial \tilde{\Omega}$, and

$$
\int_{\tilde{\Omega}} \pi \tilde{A}^{*} \Psi=\frac{1}{\gamma} \int_{\Gamma} \eta \Psi+\frac{1}{\varepsilon} \int_{\Gamma}\left(\frac{\partial \eta}{\partial n_{A^{*}}}-\rho y\right) \frac{\partial \Psi}{\partial n}
$$

$\forall \Psi$ enjoying the same properties than $\varphi$ above.

We use in (3.10)-(3.11) the weak transposed solutions of the problems involved, as in [8].

We now plug $\delta g$ for $\varphi$ (resp. $\delta \eta$ for $\Psi$ ) in (3.10) (resp. (3.11)). We obtain

$$
\delta \mathcal{J}=\int_{\tilde{\Omega}} \eta \tilde{A} \delta y+\int_{\tilde{\Omega}} \pi \tilde{A}^{*} \delta \eta+\int_{\mathcal{O}} v \delta v .
$$

Using the first variations of (3.1), we finally obtain

$$
\delta \mathcal{J}=\int_{\mathcal{O}}(\eta+v) \delta v+\int_{\omega} \eta \delta \lambda+\int_{\omega^{*}} \pi \delta \mu .
$$

One can then use the simple gradient algorithm

$$
\mid \begin{aligned}
& v^{n+1}=v^{n}-\rho\left(\eta^{n}+v^{n}\right) \\
& \lambda^{n+1}=\lambda^{n}-\rho \eta^{n} \\
& \mu^{n+1}=\mu^{n}-\rho \pi^{n}
\end{aligned}
$$

which is convergent for $\rho>0$ and small enough.

\section{REMARKS}

Remark 4.1. Let us consider again the augmented state equations (2.1)-(2.6) and let us introduce a domain decomposition

$$
\Omega=\Omega_{1} \cup \Omega_{2}
$$

with overlapping, i.e. $\Omega_{1} \cap \Omega_{2} \neq \varnothing$.

We set

$$
\Gamma_{i}=\partial \Omega_{i} \cap \partial \Omega, S_{i}=\partial \Omega_{i} \cap \Omega_{j}, j \neq i
$$

so that

$$
\partial \Omega_{i}=\Gamma_{i} \cup S_{i} .
$$

we introduce two sets

$$
\omega, \omega_{*} \subset \Omega_{1} \cap \Omega_{2}
$$

(compare to Sect. 3). We define

$$
\mid \begin{aligned}
& \chi_{i}=\text { characteristic function of } \mathcal{O} \cap \Omega_{i}\left(\chi_{i} \text { can be zero }\right) \\
& \chi, \chi_{*}=\text { characteristic function of } \omega, \omega_{*} .
\end{aligned}
$$


We now define $y_{i}, \eta_{i}$ in $\Omega_{i}$, in the following fashion:

$$
\mid \begin{aligned}
& A_{1} y_{1}=v_{1} \chi_{1}+\lambda \chi \text { in } \Omega_{1}, \\
& \frac{\partial y_{1}}{\partial n_{A_{1}}}=0 \text { on } \partial \Omega_{1}, \\
& A_{1}^{*} \eta_{1}=\mu \chi_{*} \text { in } \Omega_{1}, \\
& \frac{\partial \eta_{1}}{\partial n_{A_{1}^{*}}}=\rho y_{1} \text { on } \Gamma_{1}, 0 \text { on } S_{1} \\
& \frac{\partial y_{2}}{\partial n_{A_{2}}}=0 \text { on } \partial \Omega_{2}, \\
& A_{2}^{*} \eta_{2}=-\mu \chi_{*} \text { in } \Omega_{2}, \\
& \frac{\partial \eta_{2}}{\partial n_{A_{2}^{*}}}=\rho y_{2} \text { on } \Gamma_{2}, 0 \text { on } S_{2}
\end{aligned}
$$

where $A_{i}=$ restriction of $A$ to $\Omega_{i}$ and where $v_{1} \chi_{1}+v_{2} \chi_{2}=v 1_{\mathcal{O}}$.

If one chooses (as it is possible) the virtual controls $\lambda$ and $\mu$ in such a way that

$$
\mid \begin{aligned}
& y_{i}, \eta_{i} \text { are "approximately" } 0 \text { on } S_{i} \text { (in the } \\
& L^{2}\left(S_{i}\right) \text { topology) }
\end{aligned}
$$

then extending $y_{i}, \eta_{i}$ in $\tilde{y}_{i}, \tilde{\eta}_{i}$ by 0 outside $\Omega_{i}$, one has approximately

$$
\mid \begin{aligned}
& A\left(\tilde{y}_{1}+\tilde{y}_{2}\right)=v 1_{\mathcal{O}} \text { in } \Omega, \\
& A^{*}\left(\tilde{\eta}_{1}+\tilde{\eta}_{2}\right)=0 \text { in } \Omega, \\
& \frac{\partial}{\partial n_{A}}\left(\tilde{y}_{1}+\tilde{y}_{2}\right)=0 \text { on } \partial \Omega, \\
& \frac{\partial}{\partial n_{A^{*}}}\left(\tilde{\eta}_{1}+\tilde{\eta}_{2}\right)=\rho\left(\tilde{y}_{1}+\tilde{y}_{2}\right) \text { on } \partial \Omega .
\end{aligned}
$$

One can then proceed in a similar manner as in Section 3.2, that it is not necessary to make explicit.

Remark 4.2. The above method readily extends to the case where

$$
\Omega=\Omega_{1} \cup \ldots \cup \Omega_{N}
$$

where the covering is overlapping.

Remark 4.3. One can also apply in the present situation all the various methods introduced in [9-12] and in [5], loc.cit.

\section{REFERENCES}

[1] A. Bensoussan, J.L. Lions and R. Temam, Sur les méthodes de décomposition, de décentralisation et de coordination et applications, Méthodes Mathématiques de l'Informatique, J.L. Lions and G.I. Marchuk Eds, Dunod, Paris (1974) 133-257.

[2] I. Ekeland and R. Temam, Analyse convexe et problèmes variationnels, Dunod, Gauthier-Villars (1974).

[3] D. Gabay and J.L. Lions, Décisions stratégiques à moindres regrets. C.R. Acad. Sci. Paris 319 (1994) 1049-1056. 
[4] R. Glowinski and J.L. Lions, Exact and approximate controllability for distributed parameter systems. Acta Numer. (1994) 269-378 and (1995) 159-333.

[5] R. Glowinski, J.L. Lions and O.Pironneau, Decomposition of energy spaces and applications. C.R. Acad. Sci. Paris 329 (1999) $445-452$.

[6] J.L. Lions, Contrôle optimal des systèmes gouvernés par des équations aux dérivées partielles, Paris, Dunod, Gauthier-Villars (1968).

[7] J.L. Lions, Contrôle à moindres regrets des systèmes distribués. C.R. Acad. Sci. Paris 315 (1992) 1253-1257.

[8] J.L. Lions and E.Magenes, Problèmes aux limites non homogènes et applications, Dunod, Paris (1968) Vol. 1 and 2.

[9] J.L. Lions and O. Pironneau, Algorithmes parallèles pour la solution de problèmes aux limites. C.R. Acad. Sci. Paris 327 (1998) 947-952.

[10] J.L. Lions and O. Pironneau, Domain decomposition methods for CAD. C.R. Acad. Sci. Paris 328 (1999) 73-80.

[11] J.L. Lions and O. Pironneau, Décomposition d'opérateurs, répliques et contrôle virtuel. C.R. Acad. Sci. Paris (to appear).

[12] J.L. Lions and O. Pironneau, Sur le contrôle parallèle de systèmes distribués. C.R. Acad. Sci. Paris 327 (1998) 993-998.

[13] L.J. Savage, The foundations of Statistics, 2nd edition, Dover (1972). 